\title{
Estudo longitudinal dos fatores associados à evolução de sintomas depressivos em idosos institucionalizados
}

\author{
Longitudinal study of factors associated with the development
}

of depressive symptoms in institutionalized elderly

Filomena Vicente', Helena Espirito-Santo,2, Diana Cardoso', Fabiana da Silva', Marina Costa', Sónia Martins', Inês Torres-Pena ,2, Vera Pascoal², Fátima Rodrigues², Ana Pinto², Sara Moitinho², Sónia Guadalupe', Henrique Testa Vicente ${ }^{1,3}$, Laura Lemos ${ }^{1,2}$

\section{RESUMO}

Objetivos: Constatando que a depressão é comum em idosos institucionalizados, associando-se à solidão, à ansiedade e à afetividade, pretendemos descrever a evolução da depressão durante dois anos e verificar que fatores se associam a essa evolução. Métodos: Em um estudo de coorte prospectivo em dois momentos (2011 e 2013), avaliamos 83 idosos institucionalizados, com idade no primeiro momento entre os 60 e os 100 anos, sendo 79,5\% mulheres, 86,7\% sem companheiro(a), e 72,3\% com algum grau de escolaridade. Usamos a Escala Geriátrica da Depressão (GDS), a Escala de Solidão (UCLA-L), o Inventário Geriátrico de Ansiedade (GAl) e a Lista de Afetos Positivos e Negativos (PANAS). Resultados: Verificamos que 59,0\% mantiveram a depressão e 10,8\% desenvolveram depressão. Os idosos com depressão tiveram significativamente piores resultados na UCLA, GAI e PANAS, e os não depressivos tiveram afetos positivos mais altos. Quanto à evolução da depressão, os idosos que mantiveram depressão tiveram inicialmente pontuações elevadas no GDS, GAI, UCLA e na subescala PANAS negativo e pontuações baixas na subescala PANAS positivo. Esses idosos apresentaram associadamente um agravamento dos sentimentos de solidão, dos sintomas ansiosos e do afeto negativo ao longo dos dois anos. Os que desenvolveram depressão tiveram, no primeiro momento, pontuações elevadas na UCLA. Conclusões: Os sintomas de depressão com ou sem solidão no momento inicial, o agravamento da solidão, a ansiedade, o afeto negativo e o baixo afeto positivo poderão ser fatores de risco para a manutenção da depressão. A solidão poderá ainda ser um fator de risco para o desenvolvimento de depressão.

\section{ABSTRACT}

Objectives: Knowing that depression is common in institutionalized elderly and associated with loneliness, anxiety, and affectivity, we want to describe the evolution of depression over two years and identify which factors are associated with the development of depression. Methods: In this prospective cohort study, that encompassed two evaluation moments (2011 and 2013), 83 institutionalized elders were inquired, with age at baseline between 60 and 100 years, $79.5 \%$ women, $86.7 \%$ single and $72.3 \%$ with some type of education. The instruments

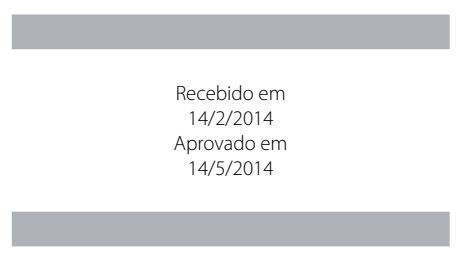

DOI: 10.1590/0047-2085000000039
1 Instituto Superior Miguel Torga, Departamento de Investigação e Desenvolvimento (DID), Coimbra, Portugal. 2 Instituto Superior Miguel Torga, Gabinete de Apoio Psicológico (GAPSI), Coimbra, Portugal.

3 Instituto Superior Miguel Torga, Núcleo João dos Santos (NJS), Coimbra, Portugal.

Endereço para correspondência: Helena Espirito Santo Instituto Superior Miguel Torga Rua Augusta, 46 3000-061 - Coimbra, Portugal E-mail: helenum@gmail.com; helenum@mac.com 


\section{Keywords}

Elderly, depression, Ioneliness, anxiety, affectivity, longitudinal. included the Geriatric Depression Scale (GDS), Loneliness Scale (UCLA-L), Geriatric Anxiety Inventory (GAl) and List of Positive and Negative Affects (PANAS). Results: Fifty-nine percent of the subjects maintained depression, and 10.8\% developed depression. Elderly patients with depression had significantly worse outcomes in the UCLA, GAI and PANAS, and those who were not depressed had a higher positive affect. Regarding the evolution of depression, elders that maintained depression had high scores on the GDS, GAl, UCLA, and negative PANAS subscale, and low scores on the PANAS positive subscale at baseline. These elders showed an increase in the feelings of loneliness, anxiety symptoms, and negative affect over the two years. Those who developed depression had higher scores on the UCLA-L at baseline. Conclusions: We conclude that depression symptoms with or without loneliness at baseline, the worsening of loneliness, anxiety, and of the positive and negative affects may all be risk factors for the maintenance of depression. Loneliness may also be a risk factor for the development of depression.

\section{INTRODUÇÃO}

A depressão é um transtorno comum em idosos ${ }^{1-3}$, aumentando a sua prevalência em idosos institucionalizados ${ }^{4-6}$.

Os fatores de risco para a depressão no idoso incluem as perdas significativas, especialmente de pessoas próximas ${ }^{1,7}$, a institucionalização ${ }^{5,8,9}$, a idade ${ }^{10,11}$ e o sexo ${ }^{12}$, embora haja estudos que não mostrem a mesma associação com a ida$\mathrm{de}^{13}$ e com o sexo ${ }^{13,14}$.

A depressão associa-se fortemente à solidão ${ }^{15,16}$ e se caracteriza por sentimentos negativos, percepção negativa da própria experiência do idoso e também pela falta/déficit de relações sociais ${ }^{6}$. Podemos ainda dizer que o estado civil tem impacto na solidão ${ }^{17}$.

A depressão associa-se também com frequência à ansiedade, com a qual se sobrepõe sintomaticamente e coenvolve $\mathrm{e}^{5,18,19}$. A sintomatologia ansiosa no idoso ocorre especialmente em alturas de transição de vida (por exemplo, a entrada na aposentadoria), de problemas de saúde física ou de perda de um dos cônjuges ${ }^{20}$. Os fatores de risco associados à ansiedade nos idosos incluem ser do sexo feminino, ter doenças crônicas, não ter companheiro, ter baixa escolaridade ${ }^{20}$, ter mais idade ${ }^{21}$ e estar institucionalizado em lar ${ }^{5,9}$.

A depressão associa-se ainda à afetividade, que é a dimensão emocional do bem-estar subjetivo. A afetividade inclui duas dimensões, a positiva e a negativa ${ }^{22}$. O afeto positivo consiste em sentimentos de alegria, de interesse, de confiança ${ }^{23}$. Em oposição, o afeto negativo é caracterizado por sofrimento subjetivo, sentimentos de raiva, de culpa, de medo, de nervosismo e por experiências desagradáveis nas atividades do cotidiano ${ }^{22}$, tristeza e desprezo ${ }^{23}$. Na depressão, estão habitualmente presentes altos níveis de afeto negativo e baixos níveis de afeto positivo ${ }^{24}$.

A revisão dos estudos longitudinais revelou como principais fatores de vulnerabilidade para a depressão em idosos residentes na comunidade (não institucionalizados) os sintomas depressivos no início da avaliação 19,25,26, os sintomas ansiosos na linha de base e um maior aumento de ansiedade ao longo do tempo ${ }^{19}$, a idade avançada ${ }^{3,26}$, o sexo feminino e a baixa escolaridade ${ }^{26}, \mathrm{o}$ acidente vascular cerebral ${ }^{27}$ e a perda de suporte social no intervalo de tempo ${ }^{25}$. Encontraram-se também evidências de que a dor e o agravamento da incapacidade se relacionam com o desenvolvimento da depressão ${ }^{25}$. Em homens, verificou-se a relação do estresse com doenças físicas e com a situação econômica; nas mulheres, o estresse relacionou-se com doenças físicas e com a solidão ${ }^{28}$.

Em síntese, a literatura mostra a alta prevalência de idosos com depressão, especialmente idosos institucionalizados. Os estudos analisados evidenciam, ainda, a relação entre a depressão e vários fatores sociodemográficos e aspectos emocionais. A revisão da literatura não revelou nenhum estudo longitudinal sobre os fatores associados à depressão em idosos institucionalizados, nem sobre fatores associados à depressão em idosos portugueses. Nesse sentido, o presente estudo teve como objetivos investigar a evolução dos sintomas depressivos avaliados pela Geriatric Depression Scale (GDS) num período de tempo de cerca de dois anos e verificar quais os fatores associados à mudança na sintomatologia depressiva.

\section{MÉTODOS}

\section{Participantes}

A partir de uma amostra de 615 idosos, incluímos 83 idosos institucionalizados em Lares ou em Centros de Dia do distrito de Coimbra, sendo 17 homens e 66 mulheres com idade igual ou superior a 60 anos, com período médio de institucionalização anterior à pesquisa de 33,90 meses ( $D P=$ $36,78)$, reavaliados com um intervalo superior a um ano $(M=$ 25,07 meses). Nenhum apresentava demência/declínio cognitivo (Montreal Cognitive Assessment $\leq 7$ pontos), acidente vascular cerebral, tumor, traumatismo craniencefálico, ou outra doença neurológica com impacto no funcionamento cognitivo, ainda que alguns idosos tivessem outras doenças médicas com potencial impacto cognitivo (Tabelas 1 e 2). 
Tabela 1. Caracterização sociodemográfica da amostra

\begin{tabular}{|c|c|c|c|c|c|c|}
\hline & \multicolumn{3}{|c|}{$\begin{array}{l}\text { Amostra elegivel } \\
\qquad n=615\end{array}$} & \multicolumn{3}{|c|}{$\begin{array}{c}\text { Amostra final } \\
n=83\end{array}$} \\
\hline & $n(\%)$ & M & DP & $\mathrm{n}(\%)$ & M & DP \\
\hline Idade & & 80,14 & 7,57 & & 79,51 & 6,58 \\
\hline \multicolumn{7}{|l|}{ Sexo } \\
\hline Homens & $148(24,1)$ & & & $17(20,5)$ & & \\
\hline Mulheres & $467(75,9)$ & & & $66(79,5)$ & & \\
\hline Total & $615(100)$ & & & $83(100)$ & & \\
\hline \multicolumn{7}{|l|}{ Estado civil } \\
\hline Sem companheiro(a) & $489(80,69)$ & & & $72(86,7)$ & & \\
\hline Com companheiro(a) & $117(19,31)$ & & & $11(13,3)$ & & \\
\hline Total & $606(100)$ & & & $83(100)$ & & \\
\hline \multicolumn{7}{|l|}{ Grau de escolaridade } \\
\hline Sem escola & $287(47,44)$ & & & $23(27,7)$ & & \\
\hline Com escola & $318(52,56)$ & & & $60(72,3)$ & & \\
\hline Total & $605(100)$ & & & $83(100)$ & & \\
\hline \multicolumn{7}{|l|}{ Profissão } \\
\hline Manual & $5(92,11)$ & & & $73(88,0)$ & & \\
\hline Intelectual & $46(7,89)$ & & & $10(12,0)$ & & \\
\hline Total & $583(100)$ & & & $83(100)$ & & \\
\hline
\end{tabular}

$n=$ número total de sujeitos; $M=$ média; $D P=$ desvio-padrão.

Tabela 2. Caracterização clínica da amostra

\begin{tabular}{lll}
\hline & $\boldsymbol{n}$ & $\%$ \\
\hline Razoavelmente saudável & 22 & 26,5 \\
DMPIC & 19 & 22,9 \\
Doença mental & 14 & 16,9 \\
Sem informação & 28 & 33,7 \\
\hline
\end{tabular}

Razoavelmente saudável: sem doenças com impacto cognitivo; DMPIC: Doença com Potencial Impacto no Funcionamento Cognitivo, incluindo esclerose múltipla, diabetes e epilepsia. Doença mental incluiu idosos diagnosticados com depressão ou com perturbação ansiosa.

\section{Medidas}

Criamos um questionário sociodemográfico para recolher informação sobre a idade, o sexo, o estado civil, a profissão e a escolaridade dos avaliados.

Avaliamos os sintomas depressivos por meio da GDS 29,30 . A GDS é constituída por 30 itens, cada um deles pontuado com zero ou um, oscilando a pontuação total entre 0 (melhor pontuação) e 30 pontos (pior pontuação). Por cada resposta afirmativa nos itens 2-4, 6, 8, 10,14, 16-18, 20, 22-26 e 28, atribui-se um ponto e por cada resposta negativa nos restantes itens, atribui-se um ponto também. As perguntas incluídas nesta escala são alusivas à última semana ${ }^{29,30}$. De acordo com os pontos de corte da escala original, um resultado entre $0 \mathrm{e}$ 10 revela ausência de depressão; um resultado entre 11 e 20 demonstra depressão ligeira e entre 21 e 30, corresponde a depressão grave ${ }^{30}$. No nosso estudo, o GDS mostrou-se com consistência interna alta $(\alpha=0,86)$, boa fidedignidade ( $r=$ $0,54, p<0,01)$ e confiabilidade $(k=0,15 ; p<0,01)$ para um intervalo de tempo médio de 14 meses $(D P=11,53)$.

A percepção de solidão foi avaliada por meio da Escala UCLA-Loneliness (UCLA-L) $)^{31,32}$. A UCLA-L é composta por 16 questões, com quatro opções de escolha que variam entre "Nunca", "Raramente", "Algumas Vezes" e "Frequentemente", com a pontuação total numa variação entre 16 e 64 pontos. Todas as perguntas foram elaboradas com um sentido negativo, devendo o sujeito referir a frequência com que apresenta sentimentos de solidão. No nosso estudo, a confiabilidade foi boa $(a=0,89)$ e o teste-reteste foi moderado $(r=0,33 ; p<0,01)$.

O Geriatric Anxiety Inventory (GAl) ${ }^{33,34}$ foi usado com objetivo de avaliar a ansiedade geriátrica. O GAl é composto por 20 itens com duas opções de resposta ("concordo" $=1$ ponto; "discordo" = 0 pontos), referindo-se à última semana. A pontuação pode oscilar entre 0 ponto (melhor pontuação) e 20

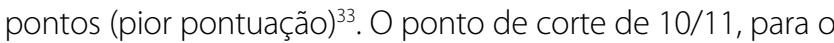
Transtorno de Ansiedade Generalizada numa amostra psicogeriátrica, apresentou sensibilidade de $75 \%$ e especificidade de $84 \%{ }^{33}$. A versão portuguesa utilizada neste estudo ${ }^{34}$ revelou confiabilidade muito boa $(a=0,93)$, validade convergente com entrevista de diagnóstico (M.I.N.I) significativa $(r=0,68 ; p$ $<0,001)$ e teste-reteste também adequado $(r=0,45 ; p<0,001)$.

Usamos a Positive and Negative Affect Schedule (PANAS $)^{22,35}$ com o objetivo de avaliar a afetividade. A PANAS é composta por 22 itens, sendo 11 deles para o afeto positivo e 11 itens para o afeto negativo. Apresentam cinco opções de resposta alusivas à última semana: "muito pouco ou nada", "um pouco", "assim, assim", "muito", "muitíssimo"35. Simões 35 constatou um alfa de Cronbach de 0,82 para a subescala de afeto positivo e de 0,85 para a subescala de afeto negativo. Constatou-se uma confiabilidade moderada para o PANAS positivo $(a=0,76)$ e para o PANAS negativo $(a=0,83)$; a fidedignidade foi moderada tanto para o PANAS positivo $(r=$ $0,39)$ quanto para o PANAS negativo $(r=0,38)$.

\section{Procedimentos}

Este estudo insere-se no Projeto Trajetórias do Envelhecimento do Instituto Superior Miguel Torga (TEISMT) iniciado em dezembro de 2010, sendo aprovado pelo Departamento de Investigação e Desenvolvimento do Instituto Superior Miguel Torga. O objetivo principal do Projeto compreendia o rastreio cognitivo e a avaliação dos idosos que se encontravam sob resposta social no distrito de Coimbra. O universo de idosos englobava 10.029 pessoas em estruturas residenciais, centros de dia/convívio e apoio domiciliário ${ }^{36}$. Das 55 instituições, 24 acederam participar, incluindo estruturas residenciais, centros de dia/convívio. A avaliação inicial ocorreu depois do consentimento informado junto de cada idoso ou dos seus cuidadores/familiares em caso de analfabetismo ou de incapacidade. As condições do estudo foram expostas a cada idoso, esclarecendo-os de que poderiam interromper 
a avaliação em qualquer altura. Os instrumentos empregados foram sempre lidos pelos avaliadores. Os dados sobre a saúde foram obtidos por meio da consulta dos processos médicos disponíveis. O processo de seleção da amostra encontra-se descrito no diagrama de decisões (Figura 1) ${ }^{37}$. Muitos idosos daquele universo não foram avaliados (92,2\%) devido a limitações nos recursos humanos e grande distância geográfica, à existência de instituições somente com idosos acamados/incapacitados/doentes, ou ainda a instituições que se recusaram a participar. Dos 781 idosos selecionados, alguns só foram avaliados em 2013, pelo que não entraram ainda no estudo longitudinal (17,0\%). Em dezembro de 2012, iniciaram-se as reavaliações. Em julho de 2013 ainda não tinham sido reavaliadas 394 pessoas e 56 pessoas não foram reavaliadas por razões variadas (recusa, doença ou morte), sendo a taxa de atrito de 20,0\%.

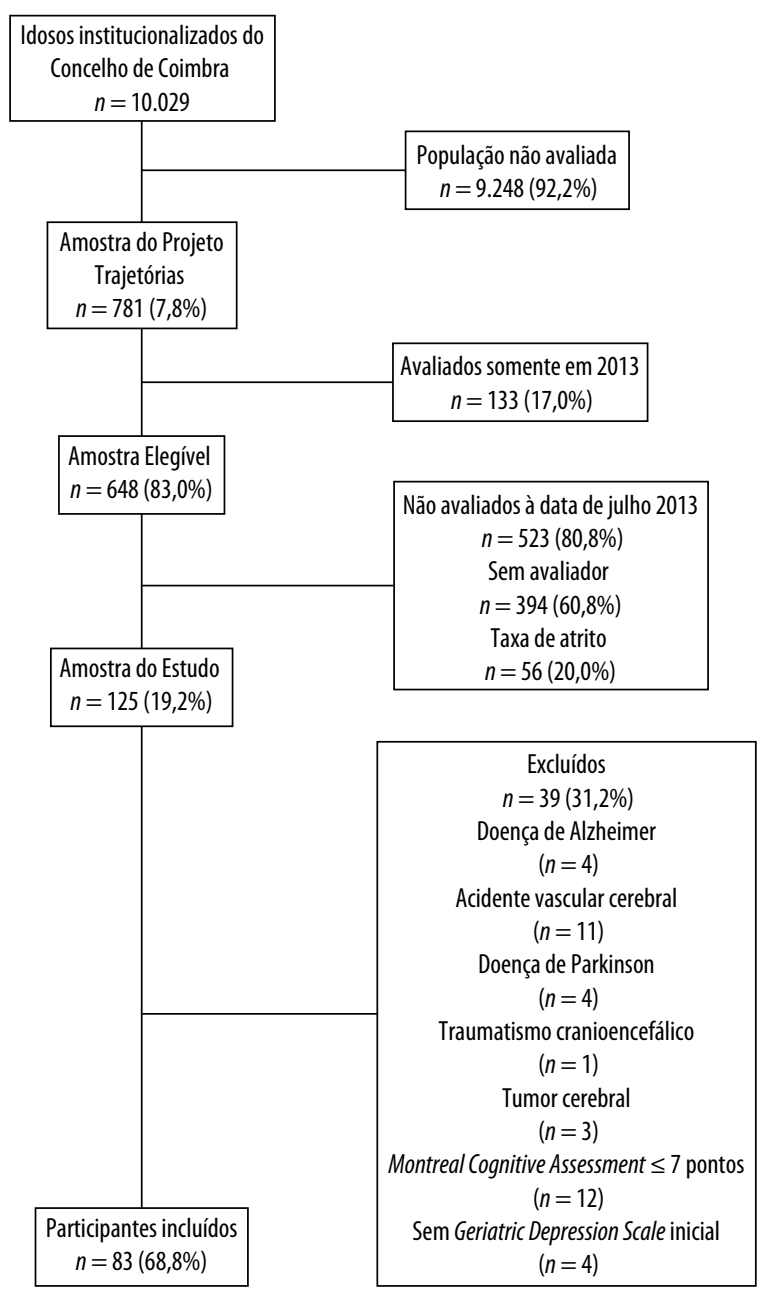

Figura 1. Diagrama de decisões do processo de inclusão de idosos no estudo. Retirado de "Estudo longitudinal dos fatores associados à evolução de sintomas depressivos em idosos institucionalizados", por F. Vicente, 2013, Coimbra, Instituto Superior Miguel Torga.
As análises foram efetuadas com o software SPSS (V.21; IBM SPSS). Para caracterizar a nossa amostra, foi realizada a estatística descritiva com o cálculo das médias, desvios-padrão, e frequências. Na análise descritiva das pontuações dos instrumentos, estudamos a normalidade da distribuição das pontuações por meio do teste de Kolmogorov-Smirnov com a correção de Lilliefors para os dois momentos. Usamos o teste $t$ para amostras emparelhadas nas variáveis emocionais. Calculamos o tamanho do efeito com a fórmula de Cumming $s^{38}$ e seguindo as indicações de Cohen ${ }^{39}$ para a sua classificação. Ainda para esse cálculo, obtivemos as correlações para amostras emparelhadas. Estudamos a evolução dos sintomas depressivos em termos de presença/ausência de depressão, categorizando as pontuações no GDS segundo os pontos de corte da versão original. Depois, consoante a evolução, determinamos as categorias seguintes: manteve-se sem depressão, melhorou, manteve depressão e desenvolveu depressão.

A seguir, em análise bivariada, fomos verificar quais os fatores demográficos e emocionais que se associavam à ausência/presença de depressão no momento inicial. Para os fatores de natureza dicotômica, usamos o teste do Quiquadrado e, para os fatores de natureza escalar, utilizamos o teste $t$. Fizemos o mesmo para o momento final, mas para as categorias manteve-se sem depressão, melhorou, manteve depressão e desenvolveu depressão. Para as comparações, usamos a ANOVA e a análise post-hoc para as variáveis de natureza escalar (GDS, UCLA-L, GAI e PANAS), e a Kruskal-Wallis para as variáveis de natureza nominal. Medimos a intensidade da relação entre a evolução da depressão e a evolução das restantes variáveis emocionais (categorizadas nos mesmos moldes de evolução da depressão), recorrendo novamente ao teste do Qui-quadrado da independência.

\section{RESULTADOS}

\section{Depressão ao longo do tempo}

Pela tabela 3 verifica-se que as mudanças nas pontuações GDS, GAl e PANAS não atingiram um nível de significância para rejeitar a hipótese nula e os tamanhos de efeito foram triviais, embora se tenha verificado maior dispersão (desvios-padrão) no segundo momento em todas as provas (exceto no PANAS negativo). Nesse âmbito, destacou-se a melhoria das pontuações da UCLA-L: apesar de não se ter obtido nível de significância para rejeitar a hipótese nula $(p>0,05)$, o tamanho de efeito foi relevante $(d=0,2)$. As correlações lineares de Pearson confirmam mudanças pouco significativas com correlações a variar entre o moderado $(r=0,4)$ e o elevado $(r=0,7)$.

Quando categorizamos a presença/ausência de depressão segundo os pontos de corte da GDS e estudamos a evolução da depressão, constatamos que 10,8\% desenvolveram 
depressão, o mesmo número de idosos melhorou, e 59,0\% mantiveram depressão, contrastando com 19,3\% que se manteve sem depressão.

\section{Fatores associados à evolução da depressão}

No momento inicial, a variável presença/ausência de depressão relacionou-se com todos os aspectos emocionais e com nenhum aspecto sociodemográfico, nem com o tempo de institucionalização (Tabela 4).

Quando analisamos os fatores demográficos da evolução da depressão (quatro categorias), usamos os resultados do teste exato obtidos pela simulação de Monte Carlo, uma vez que as condições para a análise de Qui-quadrado não se verificaram ${ }^{40}$. Observamos que não houve associação significativa entre nenhuma variável sociodemográfica e a evolução da depressão. Ainda assim, o grupo que desenvolveu depressão tinha idade um pouco mais avançada $(d \geq$
0,5), sendo maior a proporção dos idosos que não tinham escolaridade. Entre os idosos que se mantiveram com depressão houve significativamente mais sentimentos de solidão do que nos que melhoraram, mas menos do que nos que desenvolveram depressão. Verificamos, ainda, que nos idosos que se mantiveram com depressão houve mais afeto negativo e menos afeto positivo do que os que se mantiveram sem depressão. Quem desenvolveu depressão teve significativamente mais sintomas de ansiedade e menos afeto positivo do que quem se manteve sem depressão (Tabela 5).

Finalmente, comparamos a intensidade da relação entre a evolução da depressão e a evolução dos sentimentos de solidão, dos sintomas de ansiedade e dos afetos, e verificamos que os idosos que mantiveram depressão foram os que mais pioraram na solidão, nos sintomas ansiosos e nos afetos negativos (Tabela 6).

Tabela 3. Comparação das pontuações médias nos instrumentos de avaliação emocional entre dois momentos de avaliação com dois anos de intervalo

\begin{tabular}{|c|c|c|c|c|c|c|c|c|c|c|}
\hline & \multirow[t]{2}{*}{ Total } & \multicolumn{3}{|c|}{$\begin{array}{c}\text { Momento inicial } \\
(n=83)\end{array}$} & \multicolumn{3}{|c|}{$\begin{array}{c}\text { Momento final } \\
(n=83)\end{array}$} & \multirow[t]{2}{*}{$t$} & \multirow[t]{2}{*}{$d$} & \multirow[t]{2}{*}{$r$} \\
\hline & & $M$ & $D P$ & Variação & $M$ & $D P$ & Variação & & & \\
\hline GDS & 83 & 14,20 & 5,92 & $2,00-25,00$ & 13,78 & 6,38 & $0,00-29,00$ & 0,80 & 0,1 & $0,70^{* *}$ \\
\hline UCLA & 57 & 36,18 & 10,96 & $19,00-59,00$ & 33,42 & 11,98 & $16,00-60,00$ & 1,65 & $0,2^{\dagger}$ & $0,40^{* *}$ \\
\hline GAI & 83 & 12,12 & 5,85 & $0,00-20,00$ & 11,20 & 6,08 & $0,00-20,00$ & 1,40 & $0,2^{\dagger}$ & $0,50^{* *}$ \\
\hline PANAS (-) & 81 & 26,99 & 9,34 & $12,00-51,00$ & 27,47 & 8,68 & $12,00-46,00$ & $-0,44$ & 0,1 & $0,41^{* *}$ \\
\hline PANAS (+) & 81 & 27,74 & 6,29 & $12,00-40,00$ & 26,96 & 7,45 & $11,00-45,00$ & 0,92 & 0,1 & $0,40^{* *}$ \\
\hline
\end{tabular}

GDS: Geriatric Depression Scale; UCLA: Escala de Solidão; GAl: Geriatric Anxiety Inventory; PANAS: Positive and Negative Affect Schedule; M: média; DP: desvio-padrão; $t$ : teste $t$ para amostras emparelhadas; d: tamanho de efeito; $r$ : correlação de Pearson.

† Tamanho do efeito pequeno.

** Correlações significativas ao nível de 0,01 .

Tabela 4. Fatores demográficos e emocionais associados à ausência/à presença de depressão no momento inicial

\begin{tabular}{|c|c|c|c|c|}
\hline & $\begin{array}{c}\text { Sem depressão } \\
\quad(n=25)\end{array}$ & $\begin{array}{c}\text { Depressão (GDS } \geq 11) \\
\quad(n=58)\end{array}$ & $\mathrm{t} / \chi^{2}$ & $p$ \\
\hline \multicolumn{5}{|l|}{ Fatores demográficos } \\
\hline Idade $(M \pm D P)$ & $80,52 \pm 6,15$ & $79,07 \pm 6,76$ & $0,92^{\mathrm{t}}$ & 0,360 \\
\hline Sexo feminino (\%) & 72,0 & 82,8 & $1,24 \chi^{2}$ & 0,265 \\
\hline Sem companheiro (\%) & 92,0 & 84,5 & $0,86 \chi^{2}$ & 0,354 \\
\hline Sem escolaridade (\%) & 20,0 & 31,0 & $1,06 \chi^{2}$ & 0,303 \\
\hline Profissão manual (\%) & 88,0 & 87,9 & $0,00 \chi^{2}$ & 0,993 \\
\hline \multicolumn{5}{|l|}{ Fatores emocionais } \\
\hline $\mathrm{GDS}(M \pm \mathrm{DP})$ & $6,80 \pm 2,40$ & $17,40 \pm 3,69$ & $-13,17^{t}$ & 0,00 \\
\hline $\mathrm{UCLA}(M \pm \mathrm{DP})$ & $32,60 \pm 10,56$ & $38,20 \pm 10,41$ & $-1,95^{t}$ & 0,06 \\
\hline $\mathrm{GAl}(M \pm \mathrm{DP})$ & $5,56 \pm 4,37$ & $14,95 \pm 3,78$ & $-9,34^{t}$ & 0,00 \\
\hline $\operatorname{PANAS}(-)(M \pm D P)$ & $18,79 \pm 5,84$ & $30,48 \pm 8,28$ & $-7,25^{t}$ & 0,00 \\
\hline $\operatorname{PANAS}(+)(M \pm \mathrm{DP})$ & $29,92 \pm 6,91$ & $26,72 \pm 5,83$ & $1,99^{t}$ & 0,05 \\
\hline
\end{tabular}

GDS: Geriatric Depression Scale; UCLA: Escala de Solidão; GAl: Geriatric Anxiety Inventory; PANAS: Positive and Negative Affect Schedule; $t$ : teste t para amostras emparelhadas; $\chi^{2}$ : Qui-quadrado; $p$ : nível de significância estatística. 
Tabela 5. Fatores demográficos e emocionais associados à evolução da depressão

\begin{tabular}{|c|c|c|c|c|c|}
\hline & $\begin{array}{l}\text { Manteve sem depressão } \\
\qquad(n=16)\end{array}$ & $\begin{array}{l}\text { Melhorou } \\
(n=10)\end{array}$ & $\begin{array}{l}\text { Manteve depressão } \\
\quad(n=50)\end{array}$ & $\begin{array}{l}\text { Desenvolveu depressão } \\
\qquad(n=7)\end{array}$ & $\mathrm{F} / \chi^{2}$ \\
\hline \multicolumn{6}{|l|}{ Fatores demográficos } \\
\hline Idade $(M \pm D P)$ & $79,56 \pm 5,34$ & $80,90 \pm 5,63$ & $78,72 \pm 6,83$ & $83,00 \pm 8,31$ & $1,05^{\mathrm{F}}$ \\
\hline Sexo feminino (\%) & 81,3 & 60,0 & 82,0 & 85,7 & $2,72^{2 / 2}$ \\
\hline Sem companheiro (\%) & 87,5 & 100 & 82,0 & 100,0 & $3,59^{12}$ \\
\hline Sem escolaridade (\%) & 18,8 & 20,0 & 30,0 & 42,9 & $1,871^{2}$ \\
\hline Profissão manual (\%) & 81,3 & 100 & 88,0 & 85,7 & $2,08^{2}$ \\
\hline \multicolumn{6}{|l|}{ Fatores emocionais } \\
\hline$G D S(M \pm D P)$ & $5,94 \pm 2,50$ & $13,40 \pm 2,63$ & $17,74 \pm 3,99$ & $9,00 \pm 2,08$ & $52,37^{* * * \mathrm{~F}}$ \\
\hline$U C L A(M \pm D P)$ & $29,00 \pm 9,32$ & $30,25 \pm 2,81$ & $38,40 \pm 10,47$ & $39,94 \pm 12,36$ & $4,95^{\mathrm{F}}$ \\
\hline$G A I(M \pm D P)$ & $4,88 \pm 4,21$ & $12,20 \pm 4,87$ & $15,10 \pm 3,94$ & $7,29 \pm 4,54$ & $27,96^{* * * \mathrm{~F}}$ \\
\hline $\operatorname{PANAS}(-)(M \pm D P)$ & $17,00 \pm 4,42$ & $24,50 \pm 6,55$ & $30,90 \pm 8,72$ & $24,86 \pm 6,74$ & $13,13^{* * * \mathrm{~F}}$ \\
\hline $\operatorname{PANAS}(+)(M \pm D P)$ & $29,87 \pm 7,76$ & $27,30 \pm 3,56$ & $26,68 \pm 6,07$ & $30,43 \pm 6,70$ & $1,51^{\mathrm{F}}$ \\
\hline
\end{tabular}

GDS: Geriatric Depression Scale; UCLA: Escala de Solidão; GAl: Geriatric Anxiety Inventory; PANAS: Positive and Negative Affect Schedule; $F=$ Anova; $\chi^{2}=$ Qui-Quadrado; $* * * 0,008$.

Comparações significativas estatísticas ( $p<0,008)$ em análise post-hoc: GDS, Manteve sem Depressão < Melhorou; Manteve sem Depressão < Manteve Depressão; Manteve Depressão $>$ Desenvolveu Depressão; UCLA, Melhorou < Manteve Depressão; Manteve Depressão < Desenvolveu Depressão; GAl, Manteve sem Depressão < Melhorou; Manteve sem Depressão < Manteve Depressão; PANAS (-), Manteve sem Depressão < Manteve Depressão; PANAS(+), Manteve sem Depressão > Melhorou; Manteve sem Depressão > Manteve Depressão; Manteve sem Depressão > Desenvolveu Depressão.

Tabela 6. Evolução dos fatores emocionais associados à evolução da depressão

\begin{tabular}{|c|c|c|c|c|c|}
\hline & $\begin{array}{l}\text { Manteve sem depressão } \\
\qquad(n=16)\end{array}$ & $\begin{array}{l}\text { Melhorou } \\
(n=10)\end{array}$ & $\begin{array}{l}\text { Manteve depressão } \\
\quad(n=50)\end{array}$ & $\begin{array}{l}\text { Desenvolveu depressão } \\
\qquad(n=7)\end{array}$ & $\chi^{2}$ \\
\hline \multicolumn{6}{|l|}{ Fatores emocionais } \\
\hline UCLA pioria & $6,9 \%$ & $3,4 \%$ & $82,8 \%$ & $6,9 \%$ & $15,72^{* *}$ \\
\hline GAl pioria & $10,0 \%$ & $6,7 \%$ & $75,0 \%$ & $8,3 \%$ & $22,70^{* * *}$ \\
\hline PANAS (-) pioria & $9,0 \%$ & $11,9 \%$ & $71,6 \%$ & $7,5 \%$ & $27,78^{* * *}$ \\
\hline PANAS (+) pioria & $0,0 \%$ & $12,5 \%$ & $62,5 \%$ & $25,0 \%$ & 4,40 \\
\hline
\end{tabular}

UCLA: Escala de Solidão; GAl: Geriatric Anxiety Inventory; PANAS: Positive and Negative Affect Schedule; $x^{2}$ : Qui-quadrado; $p$ : nível de significância estatística.

${ }^{* *} p<0,01 ; * * * 0,001$.

\section{DISCUSSÃO}

\section{Depressão ao longo do tempo}

O presente estudo teve como objetivo compreender os fatores associados à depressão ao longo do tempo em idosos institucionalizados. Quanto aos sintomas depressivos, no início do estudo, a média foi de 14,20 $(D P=5,92)$ e, ao fim de dois anos, foi de 13,78 ( $D P=6,38)$. Podemos dizer que desceu ligeiramente do primeiro momento para o segundo $(d=0,1)$, que há uma maior dispersão no segundo momento e que existe uma correlação significativa entre os dois momentos ( $p<0,01)$. Quanto à evolução da depressão ao longo do tempo, podemos constatar que 59,0\% dos idosos mantiveram depressão, 10,8\% desenvolveram depressão e uma percentagem similar apresentou melhorias. Os nossos resultados são comparáveis aos do estudo de Harris et al. ${ }^{25}$, cujo valor de aparecimento de sintomas depressivos foi de 8,4\% em dois anos com idosos consultados em clínicas privadas. Essas semelhanças, entre amostras diferentes de populações heterogêneas, levam a pensar que o desenvolvi- mento da depressão não decorre dos desafios de saúde/econômicos/familiares/sociais próprios dessa idade. Essa dúvida talvez pudesse ser esclarecida com um estudo longitudinal controlando esses aspectos. Comparando a persistência dos sintomas depressivos com os outros estudos na comunidade, verificamos que a pesquisa de Cohen et al. ${ }^{19}$ encontrou uma persistência de 50\% em três anos e a de Solhaug et al. ${ }^{3}$ encontrou uma persistência de 54,4\% com 11 anos de intervalo. Esses achados vão no mesmo sentido que o nosso. Uma das possíveis razões para não existirem mudanças na sintomatologia depressiva é a idade mais avançada e o declínio da saúde ${ }^{25}$. Os idosos focados em nossa análise, além de institucionalizados, têm idade muito avançada e apresentam vários problemas de saúde. O fato de os idosos terem depressão no primeiro momento é um fator para continuarem deprimidos, muito provavelmente porque os idosos não são acompanhados em consultas de psiquiatria e de psicologia ou não são medicados com antidepressivos ${ }^{25}$. E isso acontecerá devido à atitude comum de se considerar a depressão como normativa na velhice. 
Relativamente aos sujeitos que apresentam melhorias, cabe assinalar que alguns estudos revelam que os motivos subjacentes à institucionalização são diversos e podem incluir dificuldades em assumir os próprios cuidados, ausência de suporte familiar, inexistência de condições de habitabilidade e falta de recursos econômicos ${ }^{41}$. Nesse sentido, é compreensível que uma elevada percentagem de idosos ingresse em instituições por sua própria iniciativa ${ }^{41}$, visando encontrar nesses ambientes algumas condições que Ihes faltavam em casa e novas oportunidades de suporte social e de acompanhamento, assim como de estabelecimento de novos laços sociais.

\section{Fatores associados à evolução da depressão}

Não encontramos associação entre a ausência/presença de sintomatologia depressiva e os fatores demográficos. A ausência de associação entre a idade e a depressão no momento inicial ( $t=0,92 ; p=0,36$ ), e no momento final do estudo $(F=1,05 ; p=0,38)$, ocorreu também no estudo longitudinal de Cohen et al..$^{19}$ e no estudo transversal de Singh e Misra ${ }^{6}$, mas não no estudo longitudinal de Solhaug et al. ${ }^{3}$, nem nos estudos transversais de Davey et al. ${ }^{10}$ e de Rothermund e Bradtstadter ${ }^{11}$. Em relação ao sexo e à depressão, não encontramos uma associação com a depressão no momento inicial do estudo $\left(X^{2}=0,44 ; p=0,51\right)$ nem no momento final $(F=$ $2,69 ; p=0,44)$, tal como aconteceu em outros estudos $8,12,18,42$. Não encontramos investigações que tenham estudado os outros fatores demográficos.

Em relação aos fatores emocionais, verificamos que os idosos com depressão têm significativamente piores resultados em todas as medidas. A solidão, tal como em outros estudos ${ }^{6,15,16}$, é mais alta nos idosos com depressão, ainda que contrariamente a outras pesquisas ${ }^{7,13,43-45}$ não haja associação entre o ter/não ter companheiro. Os sintomas de ansiedade foram também mais intensos entre os idosos com depressão, o que vai ao encontro da literatura nessa área ${ }^{5,18,19}$. $\mathrm{O}$ alto afeto negativo e o afeto positivo mais baixo entre os idosos com depressão são também consonantes com outras pesquisas que suportam o modelo tripartido da depressão $22,46,47$.

Quanto à evolução da depressão, nenhum fator demográfico se Ihe associa, em oposição aos fatores emocionais. Assim, entre os idosos que se mantêm sem depressão, verificamos que, inicialmente, têm menos sintomas depressivos do que os que melhoram ou mantêm depressão; menos sintomas ansiosos dos que melhoram e dos que mantêm depressão, menos afeto negativo do que os que mantiveram depressão e mais afeto positivo do que os que mantiveram depressão e dos que desenvolveram depressão. Os idosos que melhoraram tiveram no momento inicial menos sentimentos de solidão do que os que mantiveram depressão. Os idosos que mantiveram depressão tiveram, no momento inicial, piores pontuações nos sintomas depressivos (resultado semelhante foi encontra- do em diversas pesquisas ${ }^{19,25,26}$ e mais sentimentos de solidão do que os idosos que desenvolveram depressão. Chul-Gyu e Seungmi ${ }^{28}$ tinham também verificado que a solidão era fator de vulnerabilidade para a depressão em mulheres idosas. Sobre esses fatores, há também estudos que mostram que os sintomas ansiosos iniciais ${ }^{5,19}$, os sentimentos de solidão e a afetividade ${ }^{48}$ são fatores de risco para a depressão ${ }^{15,16}$.

Finalmente, em relação aos fatores que se relacionam com a evolução da depressão, verificamos que houve associação entre a evolução de todos os aspectos emocionais (exceto afetos positivos) e a evolução de depressão. Mais especificamente, constatamos que os idosos que mantiveram depressão foram os que mais agravaram nos sentimentos de solidão, nos sintomas ansiosos e no afeto negativo e positivo. A equipe de Cohen ${ }^{19}$ tinha também verificado essa associação com a evolução da ansiedade.

\section{Limitações}

O nosso estudo avalia os idosos em dois momentos fixos no tempo, não tendo acompanhado as mudanças da depressão que ocorreram no intervalo de tempo entre os dois momentos. Por isso, sugerimos que os aspectos emocionais sejam monitorizados com maior regularidade para compreender mais claramente quais os fatores de manutenção e/ou desenvolvimento dos sintomas depressivos. Outra limitação prende-se à amostra que não foi a mesma no momento final do estudo, por isso não podemos saber se os idosos que não foram avaliados estarão clinicamente saudáveis ou se pioraram. A razão para essa diferença prende-se, em grande medida, com a disponibilidade de recursos à altura da segunda avaliação, pois a taxa de atrito real foi somente de $20 \%$, não sendo muito diferente de outros estudos ${ }^{49}$. Finalmente, alguns instrumentos têm um nível de linguagem de difícil compreensão, principalmente para os idosos sem escolaridade, pelo que seria necessário criar versões mais apropriadas para essa população.

\section{CONCLUSÕES}

Considerando-se o que foi analisado, concluímos que ter depressão e/ou solidão em determinado momento serão fatores de risco para a persistência dos sintomas depressivos. A pioria ao longo do tempo da solidão, da ansiedade e dos afetos positivos e negativos parece também contribuir para a persistência dos sintomas depressivos. A solidão será fator de risco para o desenvolvimento da depressão.

Em face a esses resultados, consideramos que as intervenções terapêuticas individuais ou grupais poderão ajudar a tratar/prevenir os sintomas depressivos ou diminuir os sentimentos de solidão, devendo atuar-se sobre os sintomas depressivos e ansiosos e sobre a afetividade. Outra sugestão 
é a abertura dos espaços institucionais ao ambiente externo para minimizar os sentimentos de solidão.

\section{CONTRIBUIÇÕES INDIVIDUAIS}

Filomena Vicente - Recolhimento de dados, análise e interpretação dos dados, elaboração do artigo, aprovação da versão final.

Helena Espírito Santo - Concepção e desenho do estudo, análise e interpretação dos dados; elaboração e revisão do artigo, aprovação da versão final.

Henrique Vicente - Análise e interpretação dos dados; elaboração do artigo, aprovação da versão final.

Sónia Guadalupe - Análise e interpretação dos dados; revisão do artigo, aprovação da versão final.

Ana Pinto - Recolhimento de dados, análise e interpretação dos dados; revisão do artigo, aprovação da versão final.

Sónia Martins - Recolhimento de dados, análise e interpretação dos dados; revisão do artigo, aprovação da versão final.

Laura Lemos - Análise e interpretação dos dados; elaboração do artigo; aprovação da versão final.

Inês Pena - Recolhimento de dados, análise e interpretação dos dados; revisão do artigo, aprovação da versão final.

Sara Moitinho - Recolhimento de dados, análise e interpretação dos dados; revisão do artigo, aprovação da versão final.

Vera Pascoal - Recolhimento de dados, análise e interpretação dos dados; revisão do artigo, aprovação da versão final.

Fabiana Silva - Recolhimento de dados, análise e interpretação dos dados; revisão do artigo, aprovação da versão final.

Fátima Rodrigues - Recolhimento de dados, análise e interpretação dos dados; revisão do artigo, aprovação da versão final.

Diana Cardoso - Recolhimento de dados, análise e interpretação dos dados; revisão do artigo, aprovação da versão final.

Marina Costa - Recolhimento de dados, análise e interpretação dos dados; revisão do artigo, aprovação da versão final.

\section{CONFLITOS DE INTERESSE}

Helena Espírito Santo, Henrique Vicente, Sónia Guadalupe, Filomena Vicente, Laura Lemos, Sónia Martins, Inês Pena, Sara Moitinho, Fabiana Silva, Fátima Rodrigues, Vera Pascoal, Diana Cardoso, Marina Costa e Ana Pinto não possuem conflitos de interesse a serem declarados.

\section{REFERÊNCIAS}

1. lachina M, Jorgensen B, McGue M, Christensen K. A longitudinal study of depression symptomatology and widowhood in elderly Danish twins [Research Report] Sønderborg: University of Southern Denmark; 2006.

2. Torres-Pena I, Espirito-Santo H, Fermino S, Matreno J, Lemos L, Amaro H, et al. 0 impacto dos sintomas depressivos no défice cognitivo em idosos institucionalizados. In: QuevedoBlasco R, Quevedo-Blasco VG, editors. Avances en psicología clínica. Libro de capítulos del $\checkmark$ Congreso Internacional Y X Nacional de Psicología Clínica. Granada: Asociación Española de Psicología Conductual; 2012. p. 199-202.

3. Solhaug HI, Romuld EB, Romild U, Stordal E. Increased prevalence of depression in cohorts of the elderly: an 11-year follow-up in the general population - the HUNT study. Int Psychogeriatr. 2011;24(01):151-8.

4. Li YS, Meyer JS, Thornby J. Longitudinal follow-up of depressive symptoms among normal versus cognitively impaired elderly. Int J Geriatr Psychiatry. 2001;16(7):718-27.

5. Rahman TTA. Anxiety and depression in lone elderly living at their own homes and going to geriatric clubs versus those living at geriatric homes or anxiety and depression in Ione elderly. In: GAA. Health Issues around the World (archives 2006). New York: Global Action on Aging; 2006. Disponível em: http://www.globalaging.org/health/world/2006/ egyptelderly.pdf. Acesso em: 12 fev, 2014

6. Singh A, Misra N. Loneliness, depression and sociability in old age. Ind Psychiatry J. 2009:18(1):51-5.

7. Byers AL, VittinghoffE, Lui L-Y, Hoang T, Blazer DG, Covinsky KE, et al. Twenty-year depressive trajectories among older women. Arch Gen Psychiatry. 2012;69(10):1073-9.

8. Boorsma M, Joling K, Dussel M, Ribbe M, Frijters D, Marwijk HWJ. The incidence of depression and its risk factors in Dutch nursing homes and residential care homes. Am J Geriatr Psychiatry. 2012;20(11):932-42.

9. Goncalves AR, Espirito-Santo H, Matreno J, Fermino S, Guadalupe S. Declínio cognitivo, sintomas ansiosos e depressivos: estudo em idosos sob resposta social no concelho de Coimbra. In: Rodrigues A, Freitas C, editors. Problemáticas e desafios. Construção de uma nova realidade. Livro de Resumos do I Congresso de Gerontologia Social dos Açores; 2012 Abr 26-28; Angra do Heroísmo: Universidade dos Açores, Escola Superior de Enfermagem de Angra do Heroísmo. 2012. p. 119-22. acedido em 20, agosto, 2013, em http://repositorio.ismt.pt/handle/123456789/70

10. Davey A, Halverson C, Zonderman A, Costa P. Change in depressive symptoms in the Baltimore Longitudinal Study of Aging. J Gerontol B Psychol Sci Soc Sci. 2004;59B:P270-P7.

11. Rothermund K, Bradtstadter J. Coping with deficits and losses in later life: from compensatory action to accommodation. Psychol Aging. 2003;18(4):896-905.

12. Ekinci $\mathrm{M}$, Tortumluoglu G, Okanli A, Sergin S. The prevalence of depression in elderly living at home in eastern Turkey. Int J Hum Sci. 2004;1(1):1-10.

13. Blay SL, Andreoli SB, Fillenbaum GG, Gastal FL. Depression morbidity in later life: prevalence and correlates in a developing country. Am J Geriatr Psychiatry. 2007;15(9):790-9.

14. Costa E, Barreto SM, Uchoa E, Firmo JOA, Lima-Costa MF, Prince M. Prevalence of International Classification of Diseases, 10th Revision common mental disorders in the elderly in a Brazilian community: The Bambui Health Ageing Study. Am J Geriatr Psychiatry. 2007;15(1):17-27

15. Cacioppo JT, Hughes ME, Waite LJ, Hawkley LC, Thisted RA. Loneliness as a specific risk factor for depressive symptoms: cross-sectional and longitudinal analyses. Psychol Aging. 2006;21(1):140-51

16. Chlipala ML. Longitudinal study of loneliness and depression as predictors of health in mid- to later life [Dissertação]. Denton (TX): University of North Texas; 2008.

17. Weiss RS. Loneliness: the experience of emotional and social isolation. Cambridge (MA): MIT Press; 1973.

18. Borjesson-Hanson A, Waern M, Östling S, Gustafson D, Skoog I. One-month prevalence of mental disorders in a population sample of 95 -year olds. Am J Geriatr Psychiatry. 2011;19(3):284-91.

19. Cohen $\mathrm{Cl}$, Goh KH, Yaffee RA. Depression outcome among a biracial sample of depressed urban elders. Am J Geriatr Psychiatry. 2009;17(11):943-52.

20. Wolitzky-Taylor KB, Castriotta N, Lenze EJ, Stanley MA, Craske MG. Anxiety disorders in older adults: a comprehensive review. Depress Anxiety. 2010;27:190-211. 
21. Espírito-Santo H, Maia S, Matreno J, Fermino S, Torres-Pena I, Amaro H, et al. Funções executivas e sintomas de ansiedade: estudo em idosos sob resposta social. In: Cabello Domínguez LV, Ramiro MT, editors. Libro de resúmenes de los trabajos aceptados en el V Congreso Internacional y X Nacional de Psicología Clínica; 2012 Abr 26-28; Santander, Espanha. Granada: Asociación Española de Psicología Conductual. 2012. p. 553.

22. Watson D, Clark LA, Tellegen A. Development and validation of brief measures of positive and negative affect: the PANAS scales. J Pers Soc Psychol. 1988;54(6):1063-70.

23. Singh K, Jha S. Positive and negative affect, and grit as predictors of happiness and life satisfaction. J Indian Acad Appl Psychol. 2008;34:40-5.

24. Larson MJ, Kaufman DAS, Kellison IL, Schmalfuss IM, Perlstein WM. Double jeopardy! The additive consequences of negative affect on performance-monitoring decrements following traumatic brain injury. Neuropsychology. 2009;23(4):433-44.

25. Harris T, Cook DG, Victor C, DeWilde S, Beighton C. Onset and persistence of depression in older people-results from a 2-year community follow-up study. Age Ageing. 2006:35(1):25-32.

26. Wallace J, O'Hara MW. Increases in depressive symptomatology in the rural elderly: results from a cross-sectional and longitudinal study. J Abnorm Psychol. 1992;101:398-404.

27. Aben I, Lodder J, Honig A, Lousberg R, Boreas A, Verhey F. Focal or generalized vascula brain damage and vulnerability to depression after stroke: a 1-year prospective follow-up study. Int Psychogeriatr. 2006;18(01):19-35.

28. Chul-Gyu K, Seungmi P. Gender difference in risk factors for depression in communitydwelling elders. J Korean Acad Nurs. 2012;42(1):136-47.

29. Yesavage JA, Brink TL, Rose TL, Lum 0, Huang V, Adey M, et al. Development and validation of a geriatric depression screening scale: A preliminary report. J Psychiatr Res. 1983;17(1):37-49.

30. Barreto J, Leuschner A, Santos F, Sobral M. Escala de depressão geriátrica: Tradução portuguesa da Geriatric Depression Scale de Yesavage, et al. In: Grupo de Estudos de Envelhecimento Cerebral e Demência. Escalas e testes na demência. 2nd ed. Lisboa: GEECD. 2007. p. 65-7.

31. Russell D, Peplau L, Ferguson M. Developing a measure of loneliness. J Pers Assess. 1978:4:290-4

32. Pocinho M, Farate C, Dias CA. Validação psicométrica da escala UCLA-Loneliness para idosos portugueses. Interações. 2010;10(18):65-77.

33. Pachana N, Byrne G, Siddie H, Koloski N, Harley E, Arnold E. Development and validation of the Geriatric Anxiety Inventory. Int Psychogeriatr. 2007;19:103-14.

34. Espírito-Santo H, Daniel F, Lemos L, Pena I, Vicente F, Silva GF, et al. Validation of the Geriatric Anxiety Inventory (GAI) in an institutionalized elderly sample. Psychometric properties of Geriatric Anxiety Inventory (GAI) in an institutionalized elderly sample. Resumo submetido ao 16th World Congress of Psychiatry. Madrid: WPA; 2013.

35. Simões A. São os homens mais agressivos que as mulheres? Rev Port de Pedag. 1993; XXVII(3): 387-4
36. Carta Social. Rede de serviços e equipamentos do distrito de Coimbra; 2013. Disponível em: http://www.cartasocial.pt/index2.php?filtrar=hidden\&foco =cb_ distrito\&cod_distrit $0=06 \&$ cod_concelho $=0 \& \operatorname{cod}$ _freguesia $=0 \& \operatorname{cod} \_$area $=0 \& \operatorname{cod}$ valencia $=0 \& d c f=06$. Acesso em: 18 set, 2013.

37. Vicente F. Estudo longitudinal dos fatores associados à evolução de sintomas depressivos em idosos institucionalizados [dissertação]. Coimbra: Instituto Superior Miguel Torga; 2013.

38. Cummings $G$. Understanding the new statistics effect sizes, confidence intervals, and meta-analysis. New York: Routledge; 2012.

39. Cohen J. Statistical power analysis. $2^{a}$ ed. Nova Jersey: Psychology Press; 1988. p. 16-25, 283.

40. Maroco J. Análise estatística com o SPSS Statistics. 5a ed. Pero Pinheiro: Report Number; 2011.

41. Silva CF, Martins R, Santos IM, Costa J. Quality of life, family role and chronic illness in elderly people institutionalized and living at home. In: Sousa L, editor. Families in later life: Emerging themes and challenges. New York: Nova Science Publishers, Inc.; 2009. p. 135-62.

42. Roy-Byrne P, Afari N, Ashton S, Fischer M, Goldberg J, Buchwald D. Chronic fatigue and anxiety/depression: a twin study. Brit J Psychiat. 2002;180:29-34.

43. Mendes-Chiloff CL. Estudo da prevalência de sintomas depressivos e declínio cognitivo de idosos internados num Hospital de Ensino [tese]. São Paulo: Universidade Estadual Paulista; 2006.

44. Papadopoulos FC, Petridou E, Argyropoulou S, Kontaxakis V, Dessypris N, Anastasiou A, et al. Prevalence and correlates of depression in late life: a population based study from a rural Greek town. Int J Geriatr Psychiatr. 2005;20(4):350-7.

45. Vaz SF. A depressão no idoso institucionalizado: estudo em idosos residentes nos lares do distrito de Braga [dissertação]. Porto: Universidade do Porto; 2009.

46. Burns R, Anstey KJ, Windsor TD. Subjective well-being mediates the effects of resilience and mastery on depression and anxiety in a large community sample of young and middleaged adults. Aust N Z J Psychiatry. 2011;45(3):240-8.

47. Crawford JR, Garthwaite PH, Lawrie (J, Henry JD, MacDonald MA, Sutherland J, et al. A convenient method of obtaining percentile norms and accompanying interval estimates for self-report mood scales (DASS, DASS-21, HADS, PANAS, and sAD). Brit J Clin Psychol. 2009;48(2):163-80.

48. Benyamini Y, Roziner I. The predictive validity of optimism and affectivity in a longitudinal study of older adults. Pers Individ Dif. 2008:44(4):853-64.

49. Chatfield MD, Brayne CE, Matthews FE. A systematic literature review of attrition between waves in longitudinal studies in the elderly shows a consistent pattern of dropout between differing studies. J Clin Epidemiol. 2005;58(1):13-9. 